\title{
La Proximidad de los Productos Alimentarios: Turismo Gastronómico y Mercados de Abastos en la Costa Daurada (Cataluña, España) ${ }^{1}$
}

\author{
The Proximity of Food: Gastronomy Tourism and Local \\ Markets in Costa Daurada (Catalonia, Spain)
}

Francesc Fusté-Forné2 (ㅁ, F. Xavier Medina ${ }^{3}$ (1) y Lluís Mundet i Cerdan ${ }^{4}$ (c)

\section{RESUMEN}

El turismo gastronómico es una tipología turística donde los visitantes y turistas se adentran en la cultura y la naturaleza de un lugar a través de su gastronomía y patrimonio culinario. Entre las distintas posibilidades que ofrecen las prácticas de turismo gastronómico se encuentran las visitas a los mercados de abastos. Este artículo analiza el atractivo de los mercados a partir del estudio de la proximidad de los productos que se ofrecen en ellos. Para ello, se analizan tres mercados de tres localidades distintas de la Costa Daurada, en la zona sudoriental de Cataluña. Se han realizado 54 entrevistas a la totalidad de los puestos de alimentación de los mercados, y se han recopilado un total de 938 productos alimentarios. A partir del estudio de los distintos tipos de alimentos se puede determinar el potencial que ofrecen los mercados de abastos como espacios para la práctica del turismo gastronómico.

Palabras clave: autenticidad, desarrollo local, gastronomía, mercados, productos alimentarios.

\begin{abstract}
Food tourism is a tourist typology where visitors and tourists explore the culture and nature of a place through its gastronomy and culinary heritage. Visits to food markets are among the different possibilities offered by culinary tourism practices. This article analyzes the attractiveness of local food markets from the study of the proximity of their products' offer. In order to achieve this, three different markets in Costa Daurada, south-eastern Catalonia, are studied. A total of 54 interviews are carried out, including all the food selling points in the sites' markets. Thus, a total of 938 food products are collected. Data analysis shows that a third of the products have a local origin. From the study of the different types of foods it is possible to determine the potential offered by local markets as spaces to develop food tourism practices.
\end{abstract}

Key words: authenticity, local development, gastronomy, markets, food.

Recibido 22 marzo 2018; Aceptado 4 abril 2020; versión de preimpresión

Laboratori Multidisciplinar de Recerca en Turisme, Facultad de Turismo, Universitat de Girona y Cátedra UNESCO de Alimentación, Cultura y Desarrollo, Universitat Oberta de Catalunya. E-mail: researchexperiencetourism@gmail.com y francesc.fusteforne@udg.edu

Director de la Cátedra UNESCO de Alimentación, Cultura y Desarrollo, Universitat Oberta de Catalunya, España. E-mail: fxmedina@uoc.edu

Laboratori Multidisciplinar de Recerca en Turisme, Facultad de Turismo, Universitat de Girona, España. E-mail: Iluis.mundet@udg.edu 
Entre las prácticas más frecuentes asociadas al turismo gastronómico se encuentran las visitas a los mercados de productores, mercados agrícolas y mercados de abastos. Históricamente asociados al intercambio de alimentos y tradicionalmente vinculados a la compra de productos locales a pequeña escala por parte de los habitantes de un determinado lugar, los mercados han adquirido a lo largo de las últimas dos décadas un importante simbolismo como espacios para experimentar el sentido de lugar y la autenticidad de los territorios desde un punto de vista turístico (Medina \& Álvarez, 2007; Medina, 2008). Este fenómeno se ha visto impulsado por el auge del turismo gastronómico, tanto en entornos rurales y naturales como en contextos urbanos (Hjalager \& Richards, 2002; Leal, 2015). Aunque la investigación alrededor del turismo gastronómico es amplia, y desarrolla también el papel de los mercados en relación con su atractivo turístico, los estudios previos no analizan hasta qué punto los productos de los mercados de abastos son un reflejo de la identidad local y regional.

El objetivo de este artículo es, en este sentido, analizar los mercados de productos locales en tanto que espacios para la práctica del turismo gastronómico a partir del origen de sus productos, y en concreto, del grado de proximidad de esta oferta. Así, el presente estudio analiza el origen de los productos en los mercados de la Costa Daurada, destino turístico de referencia en Cataluña (Figura No 1), en la zona sudoriental (provincia de Tarragona), identificado principalmente con el turismo de sol y playa, pero con creciente influencia del turismo cultural y de negocios, destino tanto de visitantes nacionales como internacionales.

Figura $\mathrm{N}^{\circ} 1$

Mapa de ubicación de la Costa Daurada

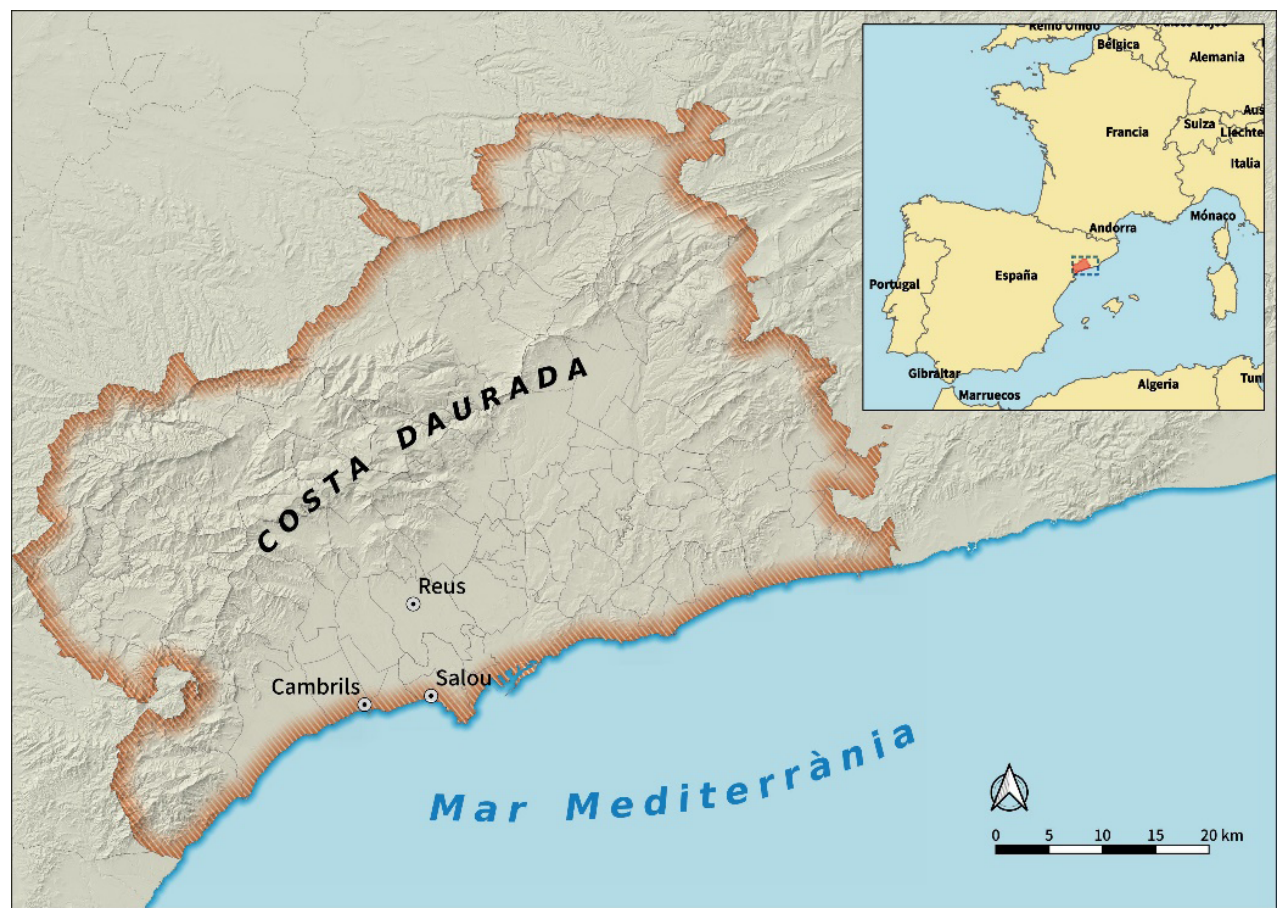

Fuente: Elaboración propia. 
A través de la investigación se determina el alcance de la distribución de los productos de proximidad, a partir de la clasificación de su tipología y origen. Para obtener una mayor fiabilidad del estudio en el contexto regional, la investigación se realiza en mercados de tres localidades distintas: Cambrils, Salou y Reus, cuya localización se puede observar en la Figura No 1 . El estudio del origen de los productos de los mercados alimentarios locales tiene implicaciones para la gestión de las relaciones entre centros de producción y distribución, y permite ver hasta qué punto los productos locales y regionales tienen o no una presencia predominante en los mercados de abastos. En consecuencia, se observa el rol de la proximidad de los productos que los visitantes y turistas adquieren durante la práctica del turismo gastronómico.

La estructura del artículo parte de una revisión de la literatura entorno a la gastronomía y el turismo, y en particular, se aproxima a los mercados de abastos como espacios para el desarrollo del turismo gastronómico. Posteriormente, se presentan las secciones de metodología y resultados, para concluir con el apartado de discusión y conclusiones, que incluyen también las limitaciones del artículo y las oportunidades de investigación futuras en el ámbito de estudio.

\section{Gastronomía, turismo y los mercados de abastos}

La gastronomía es una forma de conocimiento de la cultura y el estilo de vida de un lugar y por lo tanto ejerce una gran influencia en el sentimiento de pertenencia a un territorio (Contreras, 2004; Henderson, 2009). Como ejemplo de ello, tal y como afirman Cànoves et al. (2004), los productos frescos que se venden en los mercados de productores atraen tanto a los locales como a los turistas, ya que son un espacio que permite, a través de los alimentos, apreciar la autenticidad y las particularidades de una zona (Fusté-Forné, 2015). Esta venta de productos de proximidad permite, a través del contacto directo entre los artesanos del sector primario y los consumidores, una relación de proximidad que da un valor añadido a la venta, y a la experiencia turística.

Así mismo, esto implica un impacto económico que beneficia el desarrollo regional de los destinos, y en muchas ocasiones con especial relevancia en relación a aquellos alimentos con etiquetas de calidad (Bigné, 2011). Cuando un producto local cuenta con una de estas etiquetas, esto significa normalmente un reconocimiento a la calidad de la producción asociada a un territorio específico (Tresserras \& Medina, 2007). Las etiquetas de calidad permiten identificar el origen de los productos, lo cual investigaciones previas han desarrollado ampliamente en el contexto iberoamericano. En particular, estudios anteriores han analizado el impacto de los productos con etiquetas de calidad, principalmente la Denominación de Origen Protegida, y su relación con el turismo gastronómico (ver, por ejemplo, Millán \& Morales-Fernández, 2012). De este modo, casos como el aceite (Millán \& Agudo, 2010) o el queso (Fusté-Forné, 2018), son solo algunos de los ejemplos de productos que han sido desarrollados por la literatura con respecto a su atractivo turístico, lo cual demuestra el papel crucial del origen de los productos en el contexto de la planificación y oferta de experiencias turísticas que utilizan la gastronomía como factor de atracción.

En este contexto, la experiencia referente a las prácticas de turismo gastronómico se puede presentar de distintas maneras, siendo una de ellas las visitas a los mercados (Hall \& Sharples, 2003). Así: 
"El hecho de que se pueda disfrutar de estas actividades demuestra el interés de los turistas y visitantes en relación al origen primario de lo que comemos, a la vez que se establece una relación directa entre los anfitriones e invitados que eleva el producto gastronómico a producto turístico. Lógicamente, esta experiencia gastronómica no solo se refiere a los productores, sino también a una serie de sinergias que se generan entre las actividades agrícolas y el sector turístico. Formas de experimentarlo son los mercados de agricultores o las ferias y festivales gastronómicos, donde producto y productor se convierten en los principales protagonistas y los que transmiten la autenticidad a los visitantes. Y cuando se habla de visitantes, se incluye tanto a los propios habitantes de la región o regiones cercanas, así como a los turistas internacionales" (Fusté-Forné, 2016: 10).

Cada vez más consumidores aprecian poder conocer el origen de los productos y, especialmente, que estos sean locales, de calidad, y valorando en consecuencia su proximidad e identificación con el territorio, lo cual permite una relación auténtica y directa con la cultura local (Suárez, Huaraca \& Paladines, 2017). El turismo alimentario contribuye, a la vez, a la diversificación de la oferta del destino y a la reducción de los efectos negativos derivados de la estacionalidad de la demanda. Esto se consigue a partir de la estructuración de una oferta turística y gastronómica basada en los productos - y platos - de temporada, que cambian en función de la disponibilidad inherente a cualquier alimento, que responde a los ciclos de la naturaleza. No obstante, aquí no debe olvidarse la globalización actual y la facilidad, gracias al desarrollo de técnicas de conservación alimentaria, de disponer de productos de temporada a lo largo de todo el año.

De esta forma, el uso turístico de un producto local debe ser capaz de mantener la esencia natural y cultural de las tradiciones entorno al patrimonio culinario, y al mismo tiempo que se preserva la autenticidad local enmarcada en un entorno paisajístico específico. En consecuencia, el turismo gastronómico permite acercarse a una cultura de una manera auténtica, vivencial y participativa (Leal, 2015). A través de su papel de marcador clave de la conectividad entre culturas y lugares, la comida es uno de los recursos más importantes de cualquier destino turístico y es parte integral de este sentido de autenticidad (Fusté-Forné \& Berno, 2016).

Además, la experiencia turística también incluye el descubrimiento de los procesos de producción o recolección, y la relación compraventa con los artesanos de la alimentación, lo cual incentiva una cadena de sostenibilidad. Esta relación transmite, a la vez, el contexto cultural y el entorno natural de los productos (Bessière, 1998). Además del hecho que las experiencias gastronómicas y alimentarias locales son una parte esencial de los viajes a cualquier destino, la comida es un recurso patrimonial que adquiere cada vez más importancia como principal motivación para visitar un destino (Hall, 2016). Los visitantes perciben las herencias alimentarias como ejemplos de la identidad, con un alto valor simbólico con respecto a la importancia y el significado de un lugar (Bèssiere \& Tibère, 2013). Esto se relaciona de nuevo con la descubierta del origen de los productos, con una especial relevancia, por parte de la demanda, de aquellos que son propios del territorio donde se adquieren. Este sentido de lugar de los alimentos se puede transferir también a la industria del turismo en productos transformados en souvenirs, museos, rutas o visitas a granjas y talleres (Timothy, 2016).

Por lo tanto, los productos locales tienen un alto potencial turístico y varios usos como un factor de atracción para los destinos. Su singularidad y relevancia se comprenden cuando se 
exhiben a través de experiencias, presentando el proceso de producción e incluso permitiendo que los turistas participen, definitivamente, transmitiendo autenticidad (Byrkjeflot, Pedersen \& Svejenova, 2013). En cada contexto geográfico, esta singularidad de los productos locales se basa en un área local particular y que no puede reproducirse de igual forma en ningún otro lugar del planeta. "Los residentes y visitantes se sienten atraídos por esos lugares que han establecido un paisaje cultural y artístico único que puede utilizarse para crear una identidad de lugar distintiva" (Lee, Wall \& Kovacs, 2015: 135). Un queso Manchego 'solo' puede ser cien por cien auténtico en las zonas de producción de Castilla-la-Mancha, donde mantiene la esencia de todos sus significados - culturales y naturales, físicos y humanos -.

En este sentido, la localidad debe jugar un papel clave, gracias a movimientos como el Slow Food, que apunta a resistir la estandarización global promoviendo la gastronomía autóctona y tradicional local (Pollan, 2008). En consecuencia, se espera que las iniciativas locales puedan satisfacer las demandas de los visitantes proporcionándoles experiencias de primera mano, como escuchar historias sobre el paisaje o degustar la comida local (Jalis, Che \& Markwell, 2014), donde los comerciantes juegan un papel muy importante, tal y como se observa más adelante.

En este contexto, los mercados son lugares privilegiados para la observación de la actividad social local: un espacio público donde la gente se reúne, habla, hace circular y recibe información. Como señala Contreras, a través de los mercados "pueden pulsarse los pueblos y sus tradiciones [...]. Cualquier mercado permite una aproximación a sus pueblos, a sus culturas" (Contreras, 2004: 17-18). Son lugares de encuentro y de transacciones de gentes de diferentes orígenes y credos que han ido configurando el lugar desde un punto de vista demográfico. Lugares donde se mantiene viva, en definitiva, la relación social entre los locales. Y es precisamente esta posibilidad de sentir el pulso de una sociedad, de acercarse a sus gentes y a sus productos, la que ha favorecido, en los últimos años, que los mercados se hayan convertido, más que nunca, en un reclamo turístico de primer orden (Medina, 2008).

Por su parte, Coles \& Crang (2011: 90) afirman que:

"El mercado en sí mismo, constituido por texturas materiales distintivas y experiencias sensoriales, juega un papel importante en la apreciación de los bienes que contiene. Su localización [...]; su arquitectura; su variedad de materiales en exposición, como carretillas viejas, carros y mesas que se utilizan para mostrar productos; la multitud de gente, el ruido y el sentido de la actividad; todos estos elementos son vitales para el sentimiento y los significados del mercado. Ese sentido de lugar se traduce en una forma particular de presentación de los productos en venta, que presenta el sentido de lugar de dónde provienen estos artículos y, por lo tanto, qué cualidades tienen".

En esta dirección, un mercado alimentario es un espacio donde los productos adquieren un valor añadido como una forma de resaltar el orgullo local, y ayudar a comprender la proximidad entre producción y consumo. ¿Existe realmente una relación de proximidad? No hay estudios previos que hayan analizado esta relación a partir del origen de los productos, desde donde se construye el objetivo del presente artículo. A la vez, los mercados son lugares que contribuyen tanto el desarrollo comunitario como turístico porque implican la co-creación y la co-participación entre 
anfitriones e invitados. Así, "logran el estatus de espacios turísticos debido a sus atributos físicos, sociales, culturales - y comerciales -" (Britton, 1991: 462).

Además del papel clave de los mercados para el entorno rural, estos también emergen como espacios de gran importancia en las ciudades. De acuerdo con García (2017), el patrimonio cultural de las ciudades se representa a través de varios elementos, tanto tangibles como intangibles, entre los que se encuentran la gastronomía y, también, los mercados de abastos, que ejercen como creadores de actividad económica y de ocupación, como parte inequívoca de la cultura que alimenta la vida social de los barrios. Así, cada vez son más los turistas que visitan los mercados de abastos como un ingrediente de su experiencia gastronómica. Progresivamente, estos han ido integrando su oferta, diversificando los puntos de venta, añadiendo servicios de restauración, e incorporándose como parte de las rutas turísticas y gastronómicas de las ciudades (Crespí \& Domínguez, 2013). Es decir, no solo han ampliado horarios para hacer frente a un nuevo perfil de la demanda, sino que también han dinamizado su espacio mediante actividades y eventos culturales, y una ampliación de los servicios, que también benefician a los locales, como por ejemplo el parking o el servicio a domicilio. Como consecuencia, los mercados de abastos, pues, se han consolidado como una práctica de turismo gastronómico urbano:

"Los actuales viajeros, consideran las experiencias culinarias como un elemento clave a la hora de elegir el destino. Esa búsqueda de lo local lleva a los turistas a visitar los mercados de abastos, donde se puede percibir la idiosincrasia de la gente local, su forma de expresarse, de vender, de comprar, sus hábitos alimenticios, el estilo de vida de disfrute del ocio, los gustos y costumbres gastronómicas, etc." (García, 2017: 169).

En la misma dirección, Crespí \& Domínguez (2015) destacan que los mercados representan un atractivo turístico en los centros históricos de las ciudades:

"En un mundo globalizado, donde la homogeneización es la pauta de la cotidianeidad, aquello que busca el turista amante de nuevas experiencias es algo insólito, diferente, exclusivo, que únicamente se encuentre en aquel lugar concreto. Los monumentos, los museos, la idiosincrasia de la gente, los mercados de abastos son exponentes de lo que esos nuevos consumidores buscan, puesto que ofrecen experiencias auténticas, propias de la sociedad local que se visita. En este sentido, los mercados de abastos son uno de los recursos promocionados donde lo local y lo turístico se dan la mano" (Crespí \& Domínguez, 2015: 402).

Aunque la llegada masiva de turistas puede dificultar la convivencia, tal y como se ha estudiado en mercados como La Boqueria en Barcelona, estos permiten aumentar el nivel de ingresos de los comerciantes y, a la vez, dar una mayor visibilidad a los mercados y a las ciudades donde estos se encuentran ubicados. En ocasiones, sus céntricas ubicaciones implican un aumento de visitantes y turistas que podrían significar una pérdida de la esencia tradicional de los mercados y una cierta tendencia a la gentrificación. Por ejemplo, en un reciente reportaje de Televisió de Cataluña (programa 30 minuts, emitido el 4 de febrero de 2018) se afirmaba que debido a la masificación turística los puntos de venta que antes vendían fruta fresca ahora venden macedonia y zumos, y que aquellos que vendían pescado fresco ahora venden también cócteles de marisco y preparados. Así mismo, en el reportaje mencionado los comerciantes se llegan a preguntar si hoy verán más carros o más maletas paseando y comprando en los puestos de alimentación. 
De cualquier manera, la mayoría de ciudades construyen su vida comercial, económica y social alrededor de los mercados municipales. Y, en consecuencia, también su vida turística. Siguiendo con Crespí \& Domínguez, "los mercados ofrecen esta particularidad: poder deambular como un local, comer como uno más y formar parte de la esencia de aquel territorio social. En esta búsqueda de lo auténtico y lo local, los turistas se ven atraídos actualmente por los mercados de abastos. En estos espacios, se percibe de forma natural la idiosincrasia de la gente local, su forma de expresarse, de vender, de comprar, sus hábitos alimenticios..." (2015: 403). Por lo tanto, aunque los mercados son también un espejo de las tendencias de consumo, son concebidos como un espacio de venta auténtica, cercana, y sostenible. El objetivo de esta investigación es analizar si esto es efectivamente así.

En definitiva, la literatura muestra la clara conexión que los mercados ofrecen entre la autenticidad, la cultura local, la gastronomía, el desarrollo económico y social, y el turismo. Esta relación, para incluir la experiencia de aquello que es local y de proximidad, debe partir de una oferta de productos del territorio, lo más cercanos posible al concepto de kilómetro 0 . Ahora bien, este es un tema que las investigaciones previas han abordado escasamente, es decir, el atractivo de los mercados en relación al turismo gastronómico a partir del estudio del origen de los productos ofertados. Así mismo, esta es la principal contribución del artículo y que se desarrolla en las siguientes secciones.

\section{Metodología}

El presente artículo tiene el objetivo de estudiar el origen de los alimentos distribuidos en los mercados de productos alimentarios locales, para así analizar qué potencial ofrecen en relación a la práctica de un turismo gastronómico auténtico y local. Para ello, los mercados que se analizan como casos de estudio están ubicados en la Costa Daurada, y, en particular, en las poblaciones de Cambrils, Reus y Salou. La Costa Daurada es un destino turístico ubicado en la provincia de Tarragona, en la zona sudoriental de Cataluña. Esta es una región mediterránea donde la gastronomía está desarrollando un papel cada vez más relevante, también como parte de la evolución histórica de la alimentación, que ha desembocado, en el año 2013, en el reconocimiento por parte de la UNESCO de la dieta mediterránea como Patrimonio Inmaterial de la Humanidad.

Tal y como establece la UNESCO (2013), en este contexto, "la dieta mediterránea pone de relieve los valores de hospitalidad, buena vecindad, diálogo intercultural y creatividad, así como un modo de vida que se guía por el respeto de la diversidad". Según la misma fuente, en las geografías de la dieta mediterránea, "los mercados locales de alimentos también desempeñan un papel fundamental como espacios culturales y lugares de transmisión de la dieta mediterránea en los que la práctica cotidiana de intercambios fomenta la concordia y el respeto mutuo". Estos elementos justifican la importancia de la zona de estudio, y se añaden al dato de su relevancia turística, que se ejemplifica con las 20,1 millones de pernoctaciones en el año 2018 (Hosteltur, 2019).

En particular, el trabajo de campo y de recogida de datos se lleva a cabo durante el mes de enero de 2018. Se realizan un total de doce visitas al Mercado Municipal de Cambrils, el Mercado Central de Reus y el Mercado Municipal de Salou. En estos mercados se realizan un total de 54 entrevistas a los comerciantes con el fin de recopilar los distintos productos y su origen, y obtener la visión de los entrevistados en relación a las posibilidades turísticas de los mercados de abastos, 
que permiten articular la discusión de la investigación. Como resultado de las visitas y las entrevistas realizadas, que incluyen la totalidad de los puestos abiertos, se han recopilado un total de 938 productos alimentarios.

En relación a cada producto, se anota lo siguiente: el nombre comercial del puesto de alimentación; el tipo de establecimiento en función del producto que se ofrece; el nombre de cada alimento; su procedencia; y el ámbito de origen. En el caso de los tipos de establecimientos, estos se dividen en seis categorías distintas: carnicería/charcutería, frutería/verdulería, frutos secos, pescadería, pesca salada y pollería. En cuanto al ámbito de origen, la clasificación incluye cuatro tipos de productos de acuerdo con su procedencia: local, regional, nacional e internacional. El producto local es aquel que proviene de la propia comarca donde se encuentra el mercado, o de sus comarcas limítrofes; el producto regional es el que tiene su origen en el resto de Cataluña; el producto nacional proviene del conjunto de España; y el producto internacional es el que tiene su origen en cualquier otro país.

\section{Resultados}

Como resultado del trabajo de campo realizado se han analizado un total de 938 productos alimentarios. Estos se han dividido entre los tres mercados estudiados: Cambrils (115), Reus (668) y Salou (155). En el cuadro siguiente (Cuadro No 1) se puede observar la clasificación de los productos según el tipo de establecimiento y el lugar de recogida de los datos. De los tres mercados, el de Reus es el que incluye un mayor número de puestos de alimentación y, en consecuencia, de productos recogidos $(71,2 \%)$, muy por encima de Cambrils $(12,3 \%)$ y Salou $(16,5 \%)$.

Cuadro No 1

Clasificación de los productos según el tipo de puesto de alimentación y el mercado de abastos

\begin{tabular}{|l|r|r|}
\hline Lugar & \multicolumn{2}{|c|}{ Productos } \\
\hline Cambrils & $\mathbf{1 1 5}$ & $\mathbf{1 2 , 3 \%}$ \\
\hline Carnicería/Charcutería & 32 & $3,4 \%$ \\
\hline Frutería/Verdulería & 46 & $4,9 \%$ \\
\hline Pescadería & 30 & $3,2 \%$ \\
\hline Pesca Salada & 7 & $0,7 \%$ \\
\hline Reus & $\mathbf{6 6 8}$ & $\mathbf{7 1 , 2 \%}$ \\
\hline Carnicería/Charcutería & 174 & $18,6 \%$ \\
\hline Frutería/Verdulería & 305 & $32,5 \%$ \\
\hline Frutos secos & 19 & $2,0 \%$ \\
\hline Pescadería & 125 & $13,3 \%$ \\
\hline Pesca Salada & 27 & $2,9 \%$ \\
\hline Pollería & 18 & $1,9 \%$ \\
\hline Salou & $\mathbf{1 5 5}$ & $\mathbf{1 6 , 5 \%}$ \\
\hline Carnicería/Charcutería & 69 & $7,4 \%$ \\
\hline Frutería/Verdulería & 49 & $5,2 \%$ \\
\hline Pescadería & 32 & $3,4 \%$ \\
\hline Pollería & 5 & $0,5 \%$ \\
\hline Total & $\mathbf{9 3 8}$ & $\mathbf{1 0 0 , 0 \%}$ \\
\hline
\end{tabular}

Elaboración propia. 
El Cuadro No 1 muestra los diferentes tipos de productos en función del tipo de puesto de alimentación. De los tres mercados el de Reus es el que presenta una mayor oferta de productos, asociados a un mayor número de puestos de alimentación, y que representan más de dos tercios del total de la muestra. Esto se debe a la relación directa con la población de cada una de las tres localidades, siendo Reus la ciudad más poblada de las tres con más de 100,000 habitantes.

En general, las frutas y las verduras son los productos que presentan una mayor variedad, y, por lo tanto, un mayor peso en el total. A continuación, los alimentos de carnicería y charcutería, y la pesca fresca, significan también una parte importante de la oferta de cada mercado. Tal y como se mencionaba anteriormente, y se puede observar también en el cuadro anterior (Cuadro $N^{\circ} 1$ ), otros puestos fueron analizados, incluyendo la pesca salada, los frutos secos y las pollerías.

En relación a su procedencia, si se considera el conjunto de los alimentos, se destaca la importancia de los productos de origen local (305) y nacional (340), que predominan sobre los productos regionales (182) e internacionales (111). En este sentido, el análisis de los datos permite observar que del total de productos, el $32,52 \%$ tienen un origen local, el $19,40 \%$ un origen regional, el $36,25 \%$ son de procedencia nacional, y el 11,83\% tienen su procedencia en los mercados internacionales. En el siguiente cuadro (Cuadro $N^{\circ} 2$ ) se detallan los pesos específicos para cada uno de los tres mercados.

Cuadro $\mathrm{N}^{\circ} 2$

Clasificación de los productos según el ámbito de origen y el mercado de abastos

\begin{tabular}{|l|r|r|r|r|r|r|r|}
\hline Origen & \multicolumn{2}{|c|}{$\begin{array}{c}\text { Productos en } \\
\text { Cambrils }\end{array}$} & \multicolumn{2}{c|}{$\begin{array}{c}\text { Productos en } \\
\text { Reus }\end{array}$} & \multicolumn{2}{c|}{$\begin{array}{c}\text { Productos en } \\
\text { Salou }\end{array}$} & \multicolumn{2}{c|}{$\begin{array}{c}\text { Total } \\
\text { Productos }\end{array}$} \\
\hline Internacional & 9 & $7,8 \%$ & 84 & $12,6 \%$ & 18 & $11,6 \%$ & 311 \\
\hline Local & 58 & $50,4 \%$ & 208 & $31,1 \%$ & 39 & $25,2 \%$ & 340 \\
\hline Nacional & 35 & $30,4 \%$ & 245 & $36,7 \%$ & 60 & $38,7 \%$ & 182 \\
\hline Regional & 13 & $11,3 \%$ & 131 & $19,6 \%$ & 38 & $24,5 \%$ & $\mathbf{9 3 8}$ \\
\hline Total & $\mathbf{1 1 5}$ & $\mathbf{1 0 0 , 0 \%}$ & $\mathbf{6 6 8}$ & $\mathbf{1 0 0 , 0 \%}$ & $\mathbf{1 5 5}$ & $\mathbf{1 0 0 , 0 \%}$ & \\
\hline
\end{tabular}

Elaboración propia.

En el Cuadro $N^{\circ} 2$ se observa, pues, que el mercado de Cambrils es el que presenta una mayor oferta de productos locales. Estos, sumados a la importancia de los productos de origen regional, representan prácticamente los dos tercios de la oferta. Este mercado es el que tiene, a la vez, una menor oferta de productos de ámbito internacional, que se corresponde principalmente a piezas frutales de carácter tropical.

En relación a los productos ofertados en el mercado de Reus, aunque solo un tercio son de origen local, si se suman los de origen local y regional, ambos implican la mitad de la oferta. Los alimentos de origen nacional superan también el tercio del total. Este es el mercado con mayor presencia de productos internacionales, lo cual se debe también a un volumen de oferta y demanda mayor.

El mercado de Salou es el que tiene una oferta menor de productos locales, con solo un cuarto del total. No obstante, la suma de productos locales y regionales también representa la mitad del total de la oferta. En cambio, este mercado es el que presenta una mayor oferta de productos 
nacionales, entre los cuales destacan productos con certificaciones de calidad que están principalmente dirigidos a los turistas europeos del período estival, tal y como se observa más adelante.

A continuación, el Cuadro No 3 muestra los diferentes datos de forma conjunta. En concreto, se observa cada tipo de producto en función del mercado, y su origen, considerando la tipología de puesto de alimentación según si su origen es local, regional, nacional o internacional.

Cuadro No 3

Clasificación de los productos según el tipo de puesto de alimentación, el ámbito de origen y el mercado de abastos

\begin{tabular}{|l|r|r|r|r|r|r|r|r|}
\hline Origen/Productos & \multicolumn{2}{|c|}{ Cambrils } & \multicolumn{2}{|c|}{ Reus } & \multicolumn{2}{|c|}{ Salou } & \multicolumn{2}{|c|}{ Total } \\
\hline Internacional & $\mathbf{9}$ & $\mathbf{7 , 8} \%$ & $\mathbf{8 4}$ & $\mathbf{1 2 , 6 \%}$ & $\mathbf{1 8}$ & $\mathbf{1 1 , 6 \%}$ & $\mathbf{1 1 1}$ & $\mathbf{1 1 , 8 \%}$ \\
\hline Carnicería/Charcutería & & $0,0 \%$ & 15 & $2,2 \%$ & 8 & $5,2 \%$ & 23 & $2,5 \%$ \\
\hline Frutería/Verdulería & 8 & $7,0 \%$ & 50 & $7,5 \%$ & 9 & $5,8 \%$ & 67 & $7,1 \%$ \\
\hline Frutos secos & & $0,0 \%$ & 9 & $1,3 \%$ & & $0,0 \%$ & 9 & $1,0 \%$ \\
\hline Pescadería & & $0,0 \%$ & 6 & $0,9 \%$ & 1 & $0,6 \%$ & 7 & $0,7 \%$ \\
\hline Pesca Salada & 1 & $0,9 \%$ & 4 & $0,6 \%$ & & $0,0 \%$ & 5 & $0,5 \%$ \\
\hline Local & $\mathbf{5 8}$ & $\mathbf{5 0 , 4 \%}$ & $\mathbf{2 0 8}$ & $\mathbf{3 1 , 1 \%}$ & $\mathbf{3 9}$ & $\mathbf{2 5 , 2 \%}$ & $\mathbf{3 0 5}$ & $\mathbf{3 2 , 5 \%}$ \\
\hline Carnicería/Charcutería & 15 & $13,0 \%$ & 29 & $4,3 \%$ & 13 & $8,4 \%$ & 57 & $6,1 \%$ \\
\hline Frutería/Verdulería & 18 & $15,7 \%$ & 95 & $14,2 \%$ & 11 & $7,1 \%$ & 124 & $13,2 \%$ \\
\hline Frutos secos & & $0,0 \%$ & 3 & $0,4 \%$ & & $0,0 \%$ & 3 & $0,3 \%$ \\
\hline Pescadería & 24 & $20,9 \%$ & 63 & $9,4 \%$ & 12 & $7,7 \%$ & 99 & $10,6 \%$ \\
\hline Pesca Salada & 1 & $0,9 \%$ & 2 & $0,3 \%$ & & $0,0 \%$ & 3 & $0,3 \%$ \\
\hline Pollería & & $0,0 \%$ & 16 & $2,4 \%$ & 3 & $1,9 \%$ & 19 & $2,0 \%$ \\
\hline Nacional & $\mathbf{3 5}$ & $\mathbf{3 0 , 4 \%}$ & $\mathbf{2 4 5}$ & $\mathbf{3 6 , 7 \%}$ & $\mathbf{6 0}$ & $\mathbf{3 8 , 7 \%}$ & $\mathbf{3 4 0}$ & $\mathbf{3 6 , 2 \%}$ \\
\hline Carnicería/Charcutería & 8 & $7,0 \%$ & 86 & $12,9 \%$ & 26 & $16,8 \%$ & 120 & $12,8 \%$ \\
\hline Frutería/Verdulería & 18 & $15,7 \%$ & 107 & $16,0 \%$ & 24 & $15,5 \%$ & 149 & $15,9 \%$ \\
\hline Frutos secos & & $0,0 \%$ & 6 & $0,9 \%$ & & $0,0 \%$ & 6 & $0,6 \%$ \\
\hline Pescadería & 4 & $3,5 \%$ & 26 & $3,9 \%$ & 10 & $6,5 \%$ & 40 & $4,3 \%$ \\
\hline Pesca Salada & 5 & $4,3 \%$ & 20 & $3,0 \%$ & & $0,0 \%$ & 25 & $2,7 \%$ \\
\hline Regional & $\mathbf{1 3}$ & $\mathbf{1 1 , 3 \%}$ & $\mathbf{1 3 1}$ & $\mathbf{1 9 , 6 \%}$ & $\mathbf{3 8}$ & $\mathbf{2 4 , 5 \%}$ & $\mathbf{1 8 2}$ & $\mathbf{1 9 , 4 \%}$ \\
\hline Carnicería/Charcutería & 9 & $7,8 \%$ & 44 & $6,6 \%$ & 22 & $14,2 \%$ & 75 & $8,0 \%$ \\
\hline Frutería/Verdulería & 2 & $1,7 \%$ & 53 & $7,9 \%$ & 5 & $3,2 \%$ & 60 & $6,4 \%$ \\
\hline Frutos secos & & $0,0 \%$ & 1 & $0,1 \%$ & & $0,0 \%$ & 1 & $0,1 \%$ \\
\hline Pescadería & $1,7 \%$ & 30 & $4,5 \%$ & 9 & $5,8 \%$ & 41 & $4,4 \%$ \\
\hline Pesca Salada & $0,0 \%$ & 1 & $0,1 \%$ & & $0,0 \%$ & 1 & $0,1 \%$ \\
\hline Pollería & $\mathbf{1 1 5}$ & $\mathbf{1 0 0 , 0 \%}$ & $\mathbf{6 6 8}$ & $\mathbf{1 0 0 , 0 \%}$ & $\mathbf{1 5 5}$ & $\mathbf{1 0 0 , 0 \%}$ & $\mathbf{9 3 3}$ & $\mathbf{1 0 0 , 0 \%}$ \\
\hline Total & & & & & & & & $0,4 \%$ \\
\hline Elaboracion & & & & & & \\
\hline
\end{tabular}

Elaboración propia.

En el cuadro anterior (Cuadro $N^{\circ} 3$ ) se pueden observar los porcentajes para cada tipo de producto en función de su origen. Primero, en el caso de los productos de origen local, que, 
como se veía anteriormente, representan un tercio del total, destacan las frutas y verduras, y los productos del mar. Las frutas y verduras de temporada, pues, tienen su origen en los campos de cultivo pertenecientes a los municipios de la comarca, como Riudoms. Lo mismo sucede con los productos de pescadería, donde el puerto de Cambrils destaca como uno de los principales puntos de origen del pescado fresco.

Los alimentos con un origen regional, con un peso de casi un $20 \%$, se corresponden principalmente a productos de charcutería - con origen en las granjas de Lleida -, frutas y verduras que provienen también de comarcas de Lleida y Girona - por ejemplo productos con etiquetas de calidad como la pera de Lleida (Denominación de Origen Protegida) o la manzana de Girona (Indicación Geográfica Protegida) -. De origen regional destaca también el pescado y marisco que proviene del municipio de Sant Carles de la Ràpita, en la comarca del Montsià, en el extremo sud de Cataluña.

En relación a los productos que provienen del ámbito nacional, y que cuentan - tal y como se observa en el Cuadro No 3 anterior - con el mayor peso con poco más de un tercio del total, destacan también los productos de carnicería y charcutería. Aquí es importante mencionar principalmente los jamones y los quesos. No se puede olvidar el peso específico de las frutas y verduras nacionales, que en muchos casos no son de temporada, y que provienen del sud de España, de las comunidades autónomas de Murcia y Andalucía. Además, en cuanto al origen nacional del pescado, los comerciantes destacan los productos del Mar Cantábrico, que son abundantes en las pescaderías de los mercados municipales de abastos analizados.

Finalmente, los productos de origen internacional tienen un peso que no llega al $12 \%$ del total. El tipo de alimento con más presencia son las frutas, y, en concreto, las frutas de carácter tropical que provienen de América Central y América Latina como el caso de la piña, o de otros países como Nueva Zelanda, en el caso del kiwi. El porcentaje correspondiente a la carnicería y charcutería - relevante solo en el mercado de Salou por la mayor afluencia de turistas - incluye mayoritariamente quesos de origen internacional, provenientes de países como Francia, Holanda, o Suiza.

\section{Discusión}

Los resultados del presente estudio muestran un peso similar del binomio local-regional frente al origen nacional-internacional. Así, la suma de los productos que provienen de los ámbitos locales y regionales es de poco más de la mitad del total. Aunque esto no confirma la fuerza del producto de $\mathrm{km} \mathrm{O}$, en este sentido, la gran mayoría de los comerciantes coincidieron en afirmar que el producto viene siempre del lugar más próximo posible, y que esto es un elemento distintivo en el momento de acercar los productos a los consumidores, contando incluso algunos de los vendedores con productos provenientes de la agricultura ecológica. En consecuencia, uno de los entrevistados afirma que "los productos de proximidad son necesarios. Primero, producto de aquí, y si es posible de nuestro propio huerto. Cuando no está disponible aquí, lo traemos de fuera". Esta filosofía del producto local y de proximidad encaja con el desarrollo de la literatura existente alrededor del turismo gastronómico (ver por ejemplo volúmenes editados recientemente como Hall \& Gössling, 2016; Sloan, Legrand \& Hindley, 2015; Yeoman et al., 2015). 
No obstante, en otras ocasiones hay productos que tienen su origen en el contexto nacional e internacional debido a sus certificaciones de calidad específicas, como por ejemplo pueden ser determinados productos como cebollas, jamones ibéricos o quesos, que, como el Manchego, tienen un gran prestigio internacional. Este es también un atractivo importante para los visitantes y turistas, que no solo identifican la gastronomía como algo local de los mercados de la Costa Daurada, sino también en el contexto de Cataluña y España como destino visitado. Uno de los entrevistados destacó que "ya que aquí hay muchos turistas, todo lo que es nacional les gusta". Por ejemplo, otro comerciante afirmaba que el jamón ibérico de bellota es "lo que más vendemos".

En la misma dirección, el hecho de contar con una gran variedad de productos es también clave para la diversificación de la oferta, y poder mantener una clientela amplia, pero repetitiva en el caso de los locales. Por ejemplo, "cuantos más tipos diferentes de quesos tienes, a más gente llegas. A la mayoría de la gente le gusta el queso, son unos sibaritas del queso". Así, en esta charcutería se destaca que "durante las Navidades, tenemos también quesos internacionales que provienen de países como Holanda o Alemania, por qué a la gente le gusta, sobre todo en Navidad, adornar la mesa con cosas distintas". Uno de los ejemplos innovadores es un queso para untar de cabra con papaya. Por lo tanto, la adquisición de alimentos y de gastronomía es una muestra del gran potencial que tiene para contribuir a la diferenciación, especialización y competitividad del destino. En este sentido, los productos locales se presentan como una "ventana sensorial específica" (Telfer \& Hashimoto, 2003: 158) hacia la naturaleza de un lugar y su gente (Bessière \& Tibère, 2013).

También es muy importante la temporada de los productos. En la actualidad, aunque la mayoría de los productos están disponibles a lo largo de todo el año (Kivela \& Crotts, 2006), el origen varía en función de si es un producto de temporada o no. Como resultado de las entrevistas realizadas, destacan los ejemplos de las verduras de invierno como la alcachofa, el brócoli, las acelgas o los calçots, todos ellos de origen local. En cambio, verduras como el pimiento, la berenjena, el pepino o el tomate, tienen un origen nacional, generalmente de la zona sud de España, ya que al ser hortalizas de verano se cultivan en invernaderos durante el período invernal. En relación a las frutas y verduras con origen internacional, estos se corresponden mayoritariamente a alimentos tropicales.

El caso de los productos del mar es también muy significativo, ya que la Costa Daurada es una zona con importantes recursos marítimos y una gran industria pesquera de proximidad. Aquí, destaca el puerto pesquero de Cambrils como el más cercano, pero también el pescado fresco que proviene de las poblaciones de l'Ametlla de Mar y Sant Carles de la Ràpita, en el Delta del Ebro, que corresponde al origen regional. En cuanto a los pescados de origen nacional, los comerciantes destacaron varias piezas con origen en el Mar Cantábrico, que también se mencionaba en la sección anterior. Además, la disponibilidad del pescado depende a la vez del tiempo, pudiendo variar la oferta diaria de pescado de playa, tal y como menciona una de las entrevistadas: "aunque todo es de proximidad, hoy hace muy mal tiempo y hay poco pescado fresco".

En relación a la integración del producto del mercado de abastos con el turismo, los entrevistados coincidieron en el reto que esto supone, y que se trata de una actividad estacional ya que los turistas son un público que actúa como cliente principalmente durante el verano. Esto también es debido a que la región de la Costa Daurada es un destino tradicional de sol y playa, y, 
aunque el turismo cultural y de negocios se incentiva cada vez más - por ejemplo, en 2017 Reus actuó como Capital de la Cultura Catalana -, la estacionalidad sigue produciéndose de forma acentuada. "Los turistas vienen en verano. De Francia, Bélgica, Alemania... están más acostumbrados al mercado. El mercado siempre ha sido un centro de juntarse la gente. Vaig a fer mercat. Un punto de reunión". Otro entrevistado enfatiza que "vivimos del turismo. Los locales no van mucho al mercado, no ayudan. En Barcelona y Girona son más de ir al mercado. Aquí no tanto". Esto encaja con otra de las respuestas obtenidas: "los locales representan un porcentaje pequeño de las ventas", lo cual demuestra el peso que poco a poco han ido adquiriendo las grandes superficies comerciales para la compra habitual de productos alimentarios.

Aun así, y en relación con la práctica turística, uno de los entrevistados matiza que "debe solucionarse la poca integración con el turismo. Promocionarlo. La gente no tiene tiempo. A la gente le gusta el ocio, pasarlo bien. Los puestos bonitos, el suelo enmoquetado. Cuatro mesas y poder degustarlo al momento. Esto lo hacen en los mercados de las capitales como Barcelona y Madrid". En este sentido, es importante la conexión de los mercados locales con la promoción turística de las ciudades, y, por lo tanto, como eje estratégico de la gestión del turismo municipal y regional.

No obstante, esta integración se debe producir de una forma sostenible, no masificada, y con un gran aprecio hacia los productos, y los productores, agricultores y pescadores que hay detrás. Es la combinación de una compra sostenible y una venta responsable, porque cada vez hay una mayor preocupación por parte de la demanda en relación al origen de los productos, y la forma de obtenerlos. Y esta es la principal contribución del artículo: a partir de conocer el origen de los productos es posible entender, proyectar y promocionar los mercados como una experiencia turística que entienda la diversidad de la oferta gastronómica, y que puede ser utilizada como un factor de atracción turística (ver, por ejemplo, Mascarenhas \& Gândara, 2010). En este contexto, los vendedores son también un valor de los mercados, ya que son quienes conectan el territorio con los consumidores. Así mismo, los mercados de abastos pueden adaptarse a la práctica turística sin perder su esencia, adecuar su oferta a las nuevas costumbres (por ejemplo, la venta por Internet) y las nuevas tendencias de consumo (por ejemplo, el finger food), pero siguiendo con el papel de desarrolladores culturales, económicos y sociales de los entornos urbanos.

Finalmente, la adaptación a los consumidores - entre ellos, los turistas - también requiere una gran capacidad de especialización. En este sentido, hay productos que destacan por su singularidad, lo cual, a través de su autenticidad y vinculación con la identidad territorial, también enlaza con el atractivo turístico de los mismos (Sims, 2009). Por ejemplo, esto se observa en la $\mathrm{col}$ de paperina o la lechuga de Cuaresma, productos muy poco conocidos pero que aun pueden encontrarse en los mercados municipales gracias al esfuerzo de agricultores y vendedores por mantener alimentos de gran tradición local, y que transmiten el sabor de la tierra. Esto puede ser un atractivo tanto para locales como para visitantes, para redescubrir las raíces del lugar (Fusté-Forné \& Berno, 2016), y se manifiesta también a través de ferias y eventos temáticos alrededor de determinados productos como la alcachofa, el aceite, el queso, el vino, o la galera, también conocida como gamba imperial. La globalización, pues, debe combatirse enfatizando la singularidad de la cultura alimentaria local y su identidad, su sentido del lugar, que es un atractivo para el consumo turístico (Gyimóthy \& Mykletun, 2009; Scarpato, 2002). En este contexto, "la presencia de personas o actividades que pertenecen o se originan en el sitio puede ayudar a aumentar la confianza de los visitantes y aumentar el nivel de autenticidad percibida" (Hai Nguyen \& Cheung, 
2016: 1005), tal y como se mencionaba anteriormente. Esta mirada se puede experimentar a través de las historias de los residentes. Por ejemplo, Hjalager, Johansen \& Rasmussen (2015: 2720) sugieren que los expertos en alimentos son capaces de ofrecer historias consistentes sobre el uso y la preparación de alimentos locales. También, "la demanda expresa la necesidad de vincular los alimentos a un espacio particular, darle un origen, una historia, asociarlo con el nombre de un productor, esta búsqueda de información y proximidad con los productores es una marca de autenticidad" (Bèssiere \& Tibère, 2013: 3422).

En este sentido, "la comida local es una puerta de entrada para entender el patrimonio intangible, la cultura y la cultura alimentaria y de consumo local de un destino" (Björk \& Kauppinen-Räisänen, 2016: 180). Otros autores afirman que cuando se consumen especialidades tradicionales en su punto de origen, los visitantes muestran "un acto de complicidad con el lugar, una forma de ser parte de la intimidad de ese lugar y [...] un consumo simbólico de una tierra, una región, una provincia, su clima, su historia, su paisaje" (Bessière, 2001: 117). Así, en los mercados se ha observado la voluntad de integración con el turismo, en particular, a través de carteles en varios idiomas, tanto de bienvenida como en relación a la oferta de productos, donde se encuentran traducciones en catalán, castellano, inglés, francés y ruso. Incluso, recientemente, en el caso del mercado de Reus, se ha hecho una campaña de promoción del equipo de futbol de la ciudad - en el momento de realizar la investigación de la Segunda División española - donde el cartel promocional se realizaba en una frutería del mercado municipal. Otro ejemplo de integración con el turismo cultural se encuentra en el mercado municipal de Cambrils, en cuyas inmediaciones hay un edificio modernista que alberga el Museo Agrícola de Cambrils, que es parte del Museo de Historia de esta localidad marinera. Tampoco se puede olvidar la localización céntrica de los mercados de Reus y Salou, lo cual también facilita su conversión en espacios turísticos.

\section{Conclusiones}

Este artículo contribuye a la comprensión de los mercados como factores de atracción turística a partir del análisis del origen de sus productos, lo cual emerge como la principal implicación teórica de la investigación. Aquí, las investigaciones previas reconocen ampliamente el interés de los visitantes y turistas hacia los productos locales (ver, por ejemplo, Bèssiere \& Tibère, 2013), pero los estudios previos no han analizado de dónde provienen los productos. Además del atractivo derivado de la proximidad de los productos, pues, deben considerarse los procesos de globalización actuales que hacen inevitable que los productos locales sean también globales, y justifican la presencia de productos venidos de cualquier parte del mundo. El consumo actual es cosmopolita, y esto se refiere tanto a la configuración demográfica de la población de un lugar, resultado de continuos procesos migratorios, así como a las preferencias de los turistas, que tal y como autores como Quan \& Wang (2004) establecen, en ocasiones también prefieren encontrar 'sus' productos cuando viajan. En cualquier caso, los mercados emergen como espacios para la práctica del turismo gastronómico, donde el origen de los productos resulta un factor clave para la atracción de visitantes.

Así mismo, en la relación entre los mercados y el turismo, el diseño, planificación y promoción de la experiencia gastronómica se basa en la puesta en valor del territorio donde se origina un determinado alimento, ya que el 'paisaje' es el origen de la gastronomía (Berno, Laurin \& Maltezakis, 
2014). Tal y como se mencionaba anteriormente, esto tiene una fuerte relación con la transmisión del sentido de lugar y de la cadena de autenticidad. Entre las implicaciones prácticas, pues, este estudio aporta información a los agentes tanto del sector alimentario como de la industria turística que permite obtener una perspectiva global de cuál es el origen de los productos ofertados en la región analizada, y qué tipo de autenticidad y proximidad deriva de ello. Estos datos resultan clave para la oferta de experiencias gastronómicas en los mercados de abastos como espacios para la práctica turística.

En relación a las limitaciones de la presente investigación, ante todo, cabe decir que el estudio de los productos en otro contexto geográfico o en otra época del año podría dar lugar a unos resultados distintos. Esto es una oportunidad de investigación futura que pudiera implicar una comparativa entre destinos y entre temporadas. Otra forma de analizar el impacto de los productos locales y regionales de los mercados es el estudio de los medios de comunicación y las redes sociales (García, 2017), lo cual resulta clave para poder evolucionar en la comunicación y promoción de las visitas a los mercados como experiencias del turismo gastronómico. A la vez, es también necesario un estudio amplio de las motivaciones de la demanda (Hall, 2016) para comprobar efectivamente si entienden los productos de los mercados, y su origen, como un hecho diferencial de la oferta de turismo gastronómico de las regiones, y, en particular, de la Costa Daurada.

Financiamiento: Esta investigación ha sido posible gracias al financiamiento obtenido en la "II Convocatoria de ayudas a la movilidad de doctores RED de Campus de Excelencia Internacional con actividad agroalimentaria" de la Fundación Triptolemos y la Fundación bancaria "La Caixa".

Los autores también quieren agradecer la simpatía, dedicación y amabilidad de los entrevistados en los puestos de los mercados de Cambrils, Reus y Salou. Sin sus respuestas atentas esta investigación no habría sido posible.

\section{Referencias bibliográficas}

BERNO, T., LAURIN, U. \& MALTEZAKIS, G. The special role of agriculture in food tourism. En: WOLF, E. \& LANGE-FARIA, W. (editores). Have fork will travel: Handbook for food tourism. Portland: World Food Travel Association, 2014, p.105-114.

BESSIĖRE, J. Local development and heritage: Traditional food and cuisine as tourist attractions in rural areas. Sociologia Ruralis, 1998, Vol. 38, No 1, p. 21-34. doi: https://doi.org/10.1111/14679523.00061

BESSIĖRE, J. The role of rural gastronomy in tourism. En: ROBERTS, L. y HALL, D. (editores). Rural Tourism and Recreation: Principles to Practice. Wallingford: CABI International, 2001, p.115-118.

BESSIĖRE, J. \& TIBĖRE, L. Traditional food and tourism: French tourist experience and food heritage in rural spaces. Journal of Science of Food and Agriculture, 2013, Vol. 93, No 14, p. 3420-3425. doi: https://doi.org/10.1002/jsfa.6284 
BIGNÉ, E. Las respuestas del turista ante la imagen del lugar de origen del producto. En: FLAVIAN, C. \& FANDOS, C. (coordinadores). Turismo gastronómico: Estrategias de marketing y experiencias de éxito. Zaragoza: Prensas Universitarias, 2011, p. 51-68.

BJÖRK, P. \& KAUPPINEN-RÄISÄNEN, H. Local food: a source for destination attraction. International Journal of Contemporary Hospitality Management, 2016, Vol. 28, No 1, p. 177-194. doi: https://doi. org/10.1108/IJCHM-05-2014-0214

BRITTON, S. Tourism, capital and place: Towards a critical geography of tourism. Environment and Planning D: Society and Space, 1991, No 9, p. 457-478. doi: https://doi.org/10.1068/d090451

BYRKJEFLOT, H., PEDERSEN, J.S. \& SVEJENOVA, S. From label to practice: The process of creating new nordic cuisine. Journal of Culinary Science \& Technology, 2013, Vol. 11, No 1, p. 36-55. doi: https://doi.org/10.1080/15428052.2013.754296

CÀNOVES, G., VILLARINO, M., PRIESTLEY, G.K. \& BLANCO, A. Rural tourism in Spain: An analysis of recent evolution. Geoforum, 2004, No 35, p. 755-769.

COLES, B. \& CRANG, P. Placing Alternative Consumption: Commodity Fetishism in Borough Fine Foods Market, London. En: LEWIS, T. \& POTTER, E. (editores). Ethical Consumption. A Critical Introduction. Nueva York: Routledge, 2011, p. 87-102.

CONTRERAS, J. Mercados del Mediterráneo. En: CONTRERAS, J. (director). Mercados del Mediterráneo. Barcelona: Lundwerg, 2004.

CRESPÍ, M. \& DOMÍNGUEZ, M. Urban strategies in tourist places. Cases of Madrid and Barcelona. Rotur, Revista de Ocio y Turismo, 2013, № 6, p.13-33.

CRESPÍ, M. \& DOMÍNGUEZ, M. Los mercados de abastos y las ciudades turísticas. PASOS Revista de Turismo y Patrimonio Cultural, 2015, Vol. 14, No 2, p. 401-416. doi: https://doi.org/10.25145/j. pasos.2016.14.026

FUSTÉ FORNÉ, F. El turisme gastronòmic: autenticitat i desenvolupament local en zones rurals. Documents d'Anàlisi Geogràfica, 2015, Vol. 61, No 2, p. 289-304. doi: https://doi.org/10.5565/rev/ dag. 218

FUSTÉ FORNÉ, F. Los paisajes de la cultura: la gastronomía y el patrimonio culinario. Dixit, 2016, No 24, p. 4-16.

FUSTÉ FORNÉ, F. Alimentación y turismo: potencialidades de la elaboración de queso en España. CULTUR: Revista de Cultura e Turismo, 2018, Vol. 12, № 2, p.60-87.

FUSTÉ FORNÉ, F. \& BERNO, T. Food Tourism in New Zealand: Canterbury's Foodscapes. Journal of Gastronomy and Tourism, 2016, No 2, p. 71-86. doi: https://doi.org/10.3727/21692971 6X14720551277763 
GARCÍA, B. Los mercados de abastos y su comercialización como producto de turismo de experiencias. El caso de Madrid. Cuadernos de Turismo, 2017, № 39, p. 167-189. doi: https://doi. org/10.6018/turismo.39.290491

GYIMÓTHY, S. \& MYKLETUN, R.J. Scary food: Commodifying culinary heritage as meal adventures in tourism. Journal of Vacation Marketing, 2009, Vol. 15, No 3, p. 259-273. doi: https://doi. org/10.1177/1356766709104271

HAI NGUYEN, T.H. \& CHEUNG, C. Toward an Understanding of Tourists' Authentic Heritage Experiences: Evidence from Hong Kong. Journal of Travel and Tourism Marketing, 2016, Vol. 33, No 7, p. 999-1010. doi: https://doi.org/10.1080/10548408.2015.1075460

HALL, C.M. Heirloom products in heritage places: Farmers markets, local food, and food diversity. En: TIMOTHY, D. (editor). Heritage Cuisines: Traditions, identities and tourism. Abingdon: Routledge, 2016, p. 88-103.

HALL, C.M. \& GÖSSLING, S. Food Tourism and Regional Development: Networks, products and trajectories. Abingdon: Routledge, 2016.

HALL, C.M. \& SHARPLES, E. The consumption of experiences or the experience of consumption?: An introduction to the tourism of taste. En: HALL, C.M., SHARPLES, E., MITCHELL, R., MACIONIS, N. y CAMBOURNE, B. (editores). Food tourism around the world: Development, management and markets. Oxford: Butterworth Heinemann, 2003, p. 1-24.

HENDERSON, J.C. Food tourism reviewed. British Food Journal, 2009, Vol. 111, No 4, p. 317-326. doi: https://doi.org/10.1108/00070700910951470

HJALAGER, A.M., JOHANSEN, P.H. \& RASMUSSEN, B. Informing regional food innovation through lead user experiments: The case of blue mussels. British Food Journal, 2015, Vol. 117, No 11, p. 2706 2723. doi: https://doi.org/10.1108/BFJ-03-2015-0098

HJALAGER, A.M. \& RICHARDS, G. Tourism and gastronomy. Londres: Routledge, 2002.

HOSTELTUR. La Costa Daurada prevé una temporada turística similar a la del año pasado, 2019, disponible en https://www.hosteltur.com/comunidad/nota/019264_la-costa-daurada-preve-una-temporada-turistica-similar-a-la-del-ano-pasado.html [fecha de consulta: Noviembre 3, 2019].

JALIS, M.H., CHE, D. \& MARKWELL, K. Utilising local cuisine to market Malaysia as a tourist destination. Procedia - Social and Behavioral Sciences, 2014, No 144, p. 102-110. doi: https://doi.org/10.1016/j.sbspro.2014.07.278

KIVELA, J. \& CROTTS, J.C. Tourism and gastronomy: gastronomy's influence on how tourists experience a destination. Journal of Hospitality and Tourism Research, 2006, Vol. 30, No 3, p. 354-377. doi: https://doi.org/10.1177/1096348006286797 
LEAL, M.P. Turismo gastronómico: impulsor del comercio de proximidad. Barcelona: Editorial UOC, 2015.

LEE, A.H.J., WALL, G. \& KOVACS, J.F. Creative food clusters and rural development through place branding: Culinary tourism initiatives in Stratford and Muskoka, Ontario, Canada. Journal of Rural Studies, 2015, No 39, p.133-144. doi: https://doi.org/10.1016/j.jrurstud.2015.05.001

MASCARENHAS, R.G. \& GÂNDARA, J.M. Producción y transformación territorial. La gastronomía como atractivo turístico. Estudios y perspectivas en turismo, 2010, Vol. 19, № 5, p. 776-791.

MEDINA, F.X. Mercados urbanos en Europa: Patrimonio y promoción turística. En: ÁLVAREZ, M. \& MEDINA, F.X. (editores). Identidades en el plato. El patrimonio cultural alimentario entre Europa y América. Barcelona: Icaria, 2008, p. 207-220.

MEDINA, F.X. \& ÁLVAREZ, M. El lloc per on passa la vida... Els mercats i les demandes urbanes contemporànies (Barcelona-Buenos Aires). Revista d'Etnologia de Cataluña, 2007, № 31, p. 59-69.

MILLÁN, G. \& AGUDO, E.M. El turismo gastronómico y las Denominaciones de origen en el sur de España: Oleoturismo. Un estudio de caso 2010. PASOS Revista de Turismo y Patrimonio Cultural, 2010, Vol. 8, No 1, p. 91-112. doi: https://doi.org/10.25145/j.pasos.2010.08.008

MILLÁN, G. \& MORALES, E. Denominaciones de origen protegidas (DOP) y turismo gastronómico: una relación simbiótica en Andalucía. Gran Tour, 2012, No 6, p. 101-121.

POLLAN, M. In Defense of Food: an Eater's Manifesto. Nueva York: The Penguin Press, 2008.

QUAN, S. \& WANG, N. Towards a structural model of the tourist experience: An illustration from food experiences in tourism. Tourism management, 2004, Vol. 25, № 3, p. 297-305. doi: https:// doi.org/10.1016/S0261-5177(03)00130-4

SCARPATO, R. Gastronomy as a tourist product: The perspective of gastronomy studies. En: HJALAGER, A.M. \& RICHARDS, G. (editores). Tourism and gastronomy. Londres: Routledge, 2002, p. 51-70.

SIMS, R. Food, place and authenticity: Local food and the sustainable tourism experience. Journal of Sustainable Tourism, 2009, Vol. 17, No 3, p. 321-336. doi: https://doi. org/10.1080/09669580802359293

SLOAN, P., LEGRAND, W. \& HINDLEY, C. The Routledge Handbook of Sustainable Food and Gastronomy. Abingdon: Routledge, 2015.

SUÁREZ, J.E., HUARACA, L.E. \& PALADINES, G.V. Desarrollo turístico de los mercados municipales en centros históricos. Mercado San Francisco. Quito-Ecuador. Revista Publicando, 2017, Vol. 4, No 11, p. 331-339. 
TELFER, D.J. \& HASHIMOTO, A. Food Tourism in the Niagara Region: The Development of a NouveIle Cuisine. En: HALL, C.M., SHARPLES, E., MITCHELL, R., MACIONIS, N. \& CAMBOURNE, B. (editores), Food tourism around the world: Development, management and markets. Oxford: Butterworth Heinemann, 2003, p. 158-177.

TIMOTHY, D. Heritage Cuisines: Traditions, identities and tourism. Abingdon: Routledge, 2016.

TRESSERRAS, J.J. \& MEDINA, F.X. Patrimonio gastronómico y turismo cultural en el Mediterráneo. Barcelona: Ibertur, 2007.

UNESCO. La dieta mediterránea, 2013, disponible en https://ich.unesco.org/es/RL/la-dieta-mediterranea-00884 [fecha de consulta: Noviembre 9, 2019].

YEOMAN, Y., MCMAHON-BEATTIE, U., FIELDS, K., ALBRECHT, J.N. \& MEETHAN, K. The future of food tourism: Foodies, experiences, visions and political capital. Bristol: Channel View Publications, 2015. 
